\title{
La técnica Delphi para la creación de un dossier de programas educativos dirigidos al alumnado de origen extranjero.
}

\author{
Vanesa Ausín VillaVERdE \\ Universidad de Burgos \\ vausin@ubu.es
}

\begin{abstract}
Resumen
Este artículo versa sobre la atención educativa que se desarrolla en los centros escolares de educación primaria y educación secundaria obligatoria para la inclusión del alumnado de origen extranjero. La metodología utilizada ha sido cualitativa, mediante la Técnica Delphi. A través de esta técnica se ha construido un dossier compuesto por trece programas cuyo objetivo es la atención educativa y social a este alumnado. Para su diseño y validación han participado expertos en gestión educativa de catorce comunidades autónomas. El programa más valorado, según el grado de necesidad, ha sido la formación del profesorado.
\end{abstract}

Palabras Claves: Programas educativos, educación primaria, educación secundaria obligatoria, alumnado de origen extranjero, Técnica Delphi.

\section{Application of Delphy Method for the development of a report on educational programs adressed to foreign students.}

\begin{abstract}
This article talks about the educational assistance given in primary and secondary school for the integration of foreign students. The method used in this report is qualitative, Delphy method. Through this method we have developed a report made up of thirteen programs whose goal is the educational and social assistance to this students. Professionals in the educational area of fourteen different Autonomous regions have participated in its configuration. The best valued program in terms of level of necessity is teachers' training.
\end{abstract}

Key Words: Educational programs, primary education, secondary education compulsory, foreign pupil, Delphi Technique.

\section{Referencia normalizada:}

Ausín Villaverde, V. (2013) La técnica Delphi para la creación de un dossier de programas educativos dirigidos al alumnado de origen extranjero. Historia y Comunicación Social. Vol. 18. № Especial Diciembre. Págs. 291-304.

Sumario: 1. Introducción. 2. Objetivo de la investigación. 3. Metodología. 3.1. Primera fase. 3.2. Segunda fase. 3.2.1. Selección de expertos. 3.2.2. Técnica Delphi. 3.3. Tercera fase. 4. Resultados. 5. Conclusiones. 6. Referencias bibliográficas. 


\section{Introducción}

El tema central de esta investigación se basa en la identificación de los programas educativos necesarios para implementar la respuesta educativa adecuada en la escuela para atender a la población de origen extranjero escolarizada en los centros educativos de Primaria y Secundaria Obligatoria en España.

Este trabajo pretende acercar la realidad de la institución educativa, en relación a la atención al alumnado de origen extranjero, teniendo en cuenta el enfoque de la escuela inclusiva, Morales (2006:39)

\footnotetext{
"en un contexto inclusivo las diferencias se perciben como diferencias y no como carencias, y al alumnado diferente no se le considera un problema, sino una oportunidad más para el aprendizaje [...]"
}

Desde este punto de vista, debemos considerar que el contexto escolar se basa en la diversidad y la heterogeneidad que caracterizan las aulas, en el cual todos los agentes educativos implicados (docentes, comunidad educativa, legisladores, familias, etc.) deben adaptarse a esta situación, mediante el desarrollo de la educación intercultural e inclusiva (Carrasco, 2005 y Alcalde, 2008).

En este sentido, el enfoque intercultural en educación no se concibe solamente con los miembros de uno de los grupos culturales en contacto, sino que supone enseñar a la ciudadanía a mirar a la otra persona con una óptica distinta, para comprender como piensa, cómo siente y entender que con este tipo de educación, se obtiene un beneficio que afecta a todos los sujetos (Arjona et al, 2008).

Para conocer más de cerca la realidad del alumnado de origen extranjero, estudiaremos algunas de las necesidades que pueden presentar a su llegada al sistema educativo. Para ello seguiremos la clasificación elaborada por Ramírez (2010):

- Necesidad de adaptación lingüística, a través de la inmersión en la nueva lengua y rasgos comunicativos de la comunidad de referencia.

- Necesidad de adaptación socio-cultural en la nueva comunidad.

- Necesidad de desarrollo de sus capacidades cognitivas, motrices, lingüísticas y socioafectivas de acuerdo con una visión integral del sujeto, conforme a su edad y momento evolutivo y a través de la compensación de las posibles dificultades de desarrollo.

-Necesidad de respeto al alumno como individuo y de no discriminación a su cultura y religión de pertenencia.

-Necesidad de realizar la escolarización atendiendo a sus circunstancias, conocimientos, edad e historial académico, de modo que se pueda incorporar al curso más adecuado a sus características y conocimientos previos, con los apoyos oportunos, y de esta forma continuar con aprovechamiento su educación (LOE, Art. 78.2). 
- Necesidad de una respuesta educativa ajustada a partir de programas específicos destinados al alumnado que presenten graves carencias lingüísticas o en sus competencias o conocimientos básicos, a fin de facilitar su integración en el curso correspondiente.

Ante esta realidad en la que la diversidad es la regla (Besalú, 2002) la legislación ha contado con una serie de estrategias para afrontar estas necesidades. En la Ley Orgánica de Educación (2006) se propone que las medidas de atención a la diversidad deben ser de carácter organizativo y curricular, que permitan a los centros educativos, en el ejercicio de su autonomía, una organización flexible de las enseñanzas. Entre las medidas que se contemplarán las siguientes:

- Adaptaciones del currículum. Este concepto debe ser entendido como una estrategia de planificación y actuación docente que trata de responder a las necesidades de formación que plantean determinados estudiantes, previa identificación y valoración de las mismas. Su objetivo es servir de guía al profesorado para la toma de decisiones con respecto a qué es lo que el alumnado debe aprender, cómo y cuándo lo aprenderá y cómo se evaluará su proceso de enseñanza-aprendizaje (Fernández Fernández, 2010).

- Agrupamientos flexibles que tienen como objetivo convertirse en medio educativo que, bajo la aplicación de diversas combinaciones de criterios, reestructura el grupo/clase de referencia, adscribiendo los alumnos a diversos tipos de grupos, los cuales se configuran flexiblemente en razón de las necesidades educativas de sus componentes y de su evolución en el tiempo (Oliver Vera, 2003).

- Otras medidas puestas en marcha son la optatividad de las materias, el desdoblamiento de grupos, los programas de refuerzo o los programas de tratamiento personalizado.

Por tanto que el fenómeno de la inmigración supone, desde un punto de vista intercultural, una enorme riqueza humana y, desde el punto educativo, un reto que exige dos importantes innovaciones dentro del sistema educativo actual: la innovación de las metodologías pedagógicas y la innovación de las organizaciones escolares.

En este sentido, creemos correcta la afirmación de Fernández Batanero (2006:6) cuando apunta de que la

“existencia de soluciones mágicas que eliminen los posibles problemas es pura utopía, pero una cosa sí sabemos, que teniendo en cuenta una serie de actuaciones y principios educativos estaremos cada vez más cerca de responder a las necesidades de las personas. La educación en contextos multiculturales es una realidad, y debemos de adecuar nuestras actuaciones. La utopía nos tiene que servir para seguir caminando en la dirección de la inclusión educativa". 


\section{Objetivo de investigación}

El objetivo general de esta investigación ha sido la creación y validación de un dossier formado por programas educativos destinados al alumnado de origen extranjero en Educación Primaria y Educación Secundaria Obligatoria.

\section{Metodología}

La metodología utilizada ha sido cualitativa y el actor metodológico principal la técnica Delphi. Para la consecución del objetivo planteado, la investigación se ha diseñado en tres fases (Figura 1).

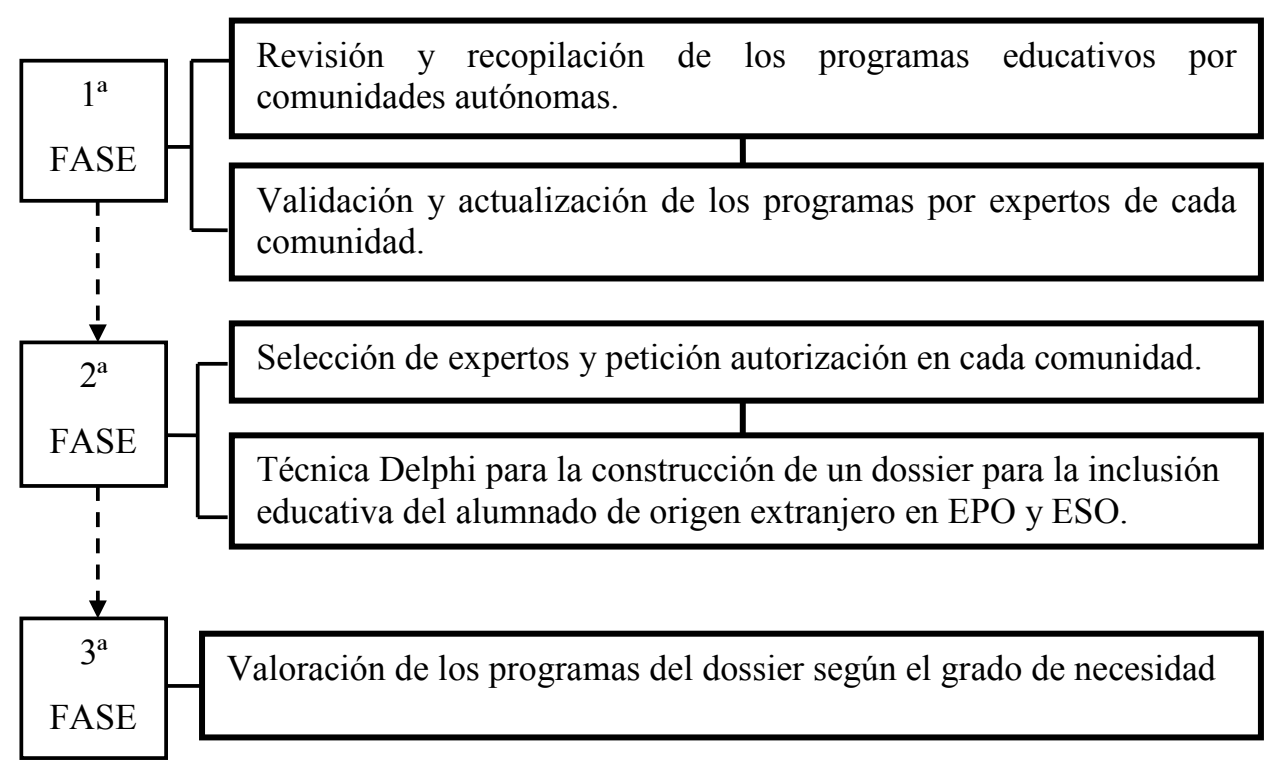

Figura 1. Fases de investigación.

\subsection{Primera fase}

Las tareas desarrolladas en esta etapa del estudio han sido:

- Identificación de los programas educativos dirigidos al alumnado de origen extranjero en todas las comunidades. Para ello se han revisado tres fuentes de información: el Centro de Recursos para la Atención a la Diversidad Cultural en Educación 
(CREADE), las páginas web de las Consejerías de Educación de cada comunidad y el estudio del Centro de Investigación y Documentación Educativa (CIDE) titulado "La atención al alumnado inmigrante en el sistema educativo en España" (2005).

- Tras la recopilación de los programas en las fuentes descritas anteriormente, se ha procedido a la validación de la misma. Para ello se ha contado con profesionales, expertos y gestores de cada comunidad autónoma. Estos profesionales se han encargado de añadir, modificar o eliminar la información extraída en el paso anterior. Por ello, puede concluirse que al finalizar esta fase se ha contado con una actualización del estudio realizado por el CIDE (2005).

En esta fase se obtuvo la participación de dieciséis comunidades.

\subsection{Segunda Fase}

Concluido el proceso de revisión y validación de los programas educativos en la primera fase se inicia la puesta en marcha de la técnica Delphi para la construcción de un dossier que recoja los programas educativos presentes en todas las comunidades.

El documento de trabajo presenta las siguientes características:

Se ha elaborado a partir del conjunto de programas de inclusión educativa validados por cada comunidad en la primera fase.

Algunos de estos programas, tienen que entenderse en el marco general de planificación y organización educativa.

Los nombres de cada recurso o programa son genéricos, para evitar la asignación directa a una u otra comunidad.

\subsubsection{Selección de expertos}

Según Ruiz Olabuénaga (1999) un muestreo no aleatorio intencional o de juicio es aquel en que la lógica y el sentido común pueden usarse para seleccionar la muestra que represente a una determinada población o situación. Por ejemplo, en la selección de expertos para la técnica Delphi (Moráguez, 2006).

Las personas que han participado debían cumplir los siguientes criterios:

Pertenecer a diferentes comunidades autónomas

Conocer la realidad educativa de este alumnado y de sus necesidades

Experiencia en cuanto a los recursos y programas educativos dirigidos al alumnado de origen extranjero escolarizado en educación primaria y educación secundaria

La petición de autorización e información se realizó a través de correo electrónico y se obtuvo la colaboración de catorce comunidades. 


\subsubsection{Técnica Delphi}

La Técnica Delphi ha constado de un total de cuatro envíos, a través de los cuales se ha ido construyendo el dossier. Durante este proceso, el documento inicial elaborado ha ido enriqueciéndose y adoptando la estructura necesaria para que pueda servir de orientación a todas las comunidades, a la hora de organizar y planificar los programas de atención educativa a este alumnado. Veamos resumidamente el proceso desarrollado:

- Envío 1. Emisión al grupo de expertos el índice de los programas que forman el documento inicial (doce programas). La función de este envío fue la valoración de la pertinencia, la eliminación o la modificación de alguno de los programas que se recogían.

- Envío 2. Para facilitar la lectura del documento, se decidió dividir la información en diferentes envíos y por lo tanto separar el documento en partes. En este momento se mandó al panel de expertos cuatro programas junto con su descripción, principios, objetivos, metodología, etc. Una vez recibidas las aportaciones se re-envió de nuevo el documento con toda la información recogida.

- Envío 3. Compuesto por el resto de programas que componen el documento. El objetivo es que cada experto añada, modifique o elimine la información que le parezca pertinente, para enriquecer cada programa. Tras el análisis de la información obtenida en este envío, se generó un nuevo programa: alumnado mentor de acogida.

- Envío 4. Ha consistido en la recopilación de todos los programas (trece en total) recogidos en los envíos anteriores con las aportaciones que se han recibido. La información aportada se ha analizado teniendo en cuenta la globalidad del documento e intentando salvar las especificidades propias de cada comunidad. En este envío se han perfilado los aspectos y los detalles específicos que forman el dossier de consenso.

\subsubsection{Tercera fase}

Con el documento de consenso definitivo, se solicitó al equipo colaborador la valoración de los programas. Esta se ha realizado en una escala tipo Likert del 1 al 5, según el grado de importancia de cada programa en relación a las necesidades educativas de este alumnado y del sistema educativo.

Tras la valoración se han extraído las puntuaciones medias para poder establecer la posición que cada recurso ocupa dentro del documento global. Estas puntuaciones han generado el siguiente orden de prioridad:

- Fundamental: estos programas deben ser implementados por todas las comunidades autónomas dentro del ámbito educativo.

- Importante: cada comunidad valorará su implantación en cada centro escolar, aunque deberá darse una alta prioridad de desarrollo. Para realizar esta valoración 
se deberán tener en cuenta criterios como: tasa de alumnos de origen extranjero en relación con alumnado de origen nacional, profesorado, otros recursos de apoyo, etc.

- De interés: los programas incluidos en este nivel, siendo relevantes, deberán ser valorados por los centros para su desarrollo, en relación a sus medios económicos, profesionales, materiales...

\section{Resultados}

El dossier de programas educativos dirigido al alumnado de procedencia extranjera está formado por 13 programas, con el objetivo de responder a las necesidades educativas y sociales del alumnado, el profesorado, las familias y la comunidad educativa.

Como puede observarse en la Figura 2 tenemos el nombre de los programas que componen el dossier y la valoración obtenida según el grado de necesidad.

\begin{tabular}{|c|c|c|c|}
\hline & Programas & Puntuación media & Valoración \\
\hline Programa 1 & Plan de formación para el profesorado & 4.78 & Fundamental \\
\hline Programa 2 & $\begin{array}{l}\text { Plan de acogida e inclusión al alumnado de } \\
\text { origen extranjero en el centro educativo }\end{array}$ & 4.71 & Fundamental \\
\hline Programa 3 & $\begin{array}{l}\text { Plan marco de acogida e inclusión al } \\
\text { alumnado y las familias de origen extranjero } \\
\text { en el sistema educativo }\end{array}$ & 4.57 & Fundamental \\
\hline Programa 4 & Programa de apoyo lingüístico & 4.5 & Fundamental \\
\hline Programa 5 & $\begin{array}{l}\text { Tutor de acogida y/o coordinador/a } \\
\text { intercultural }\end{array}$ & 4.28 & Importante \\
\hline Programa 6 & Programa de enlace con el entorno & 4.23 & Importante \\
\hline Programa 7 & Programa de mediación intercultural & 4.21 & Importante \\
\hline Programa 8 & \begin{tabular}{|l|} 
Programa para el conocimiento y \\
mantenimiento de la lengua y cultura de \\
origen del alumnado de procedencia extranjera
\end{tabular} & 4.14 & Importante \\
\hline Programa 9 & $\begin{array}{l}\text { Centros de recursos para trabajar la educación } \\
\text { intercultural }\end{array}$ & 4.07 & Importante \\
\hline Programa 10 & Alumnado mentor de acogida & 3.92 & De interés \\
\hline Programa 11 & Servicio de traducción e interpretación & 3.78 & De interés \\
\hline
\end{tabular}




\begin{tabular}{|l|l|c|c|}
\hline Programa 12 & $\begin{array}{l}\text { Cartelería en las distintas dependencias del } \\
\text { centro }\end{array}$ & 3.42 & De interés \\
\hline Programa 13 & $\begin{array}{l}\text { Guía de información multilingüe del sistema } \\
\text { educativo }\end{array}$ & 3.35 & De interés \\
\hline
\end{tabular}

Figura 2. Programas que forman el dossier y valoración de los mismos.

Analicemos el contenido de cada programa de forma resumida para comprender el sentido del dossier construido. Para conocer todo el documento puede consultarse Ausín y Lezcano (2011).

\section{Programa 1. Plan de formación para el profesorado.}

Este programa contempla realizar formación sobre educación intercultural e inclusiva para el profesorado en general y sobre la intervención educativa y el aprendizaje lingüístico y curricular para el profesorado implicado directamente con el alumnado de origen extranjero. Esta formación se orientará, entre otros aspectos hacia la metodología y didáctica para la enseñanza del castellano o lenguas de las comunidades autónomas, estrategias para la acogida, claves culturales, interculturalidad (prevención y resolución de conflictos, convivencia)...

Programa 2. Plan de acogida e inclusión al alumnado y las familias de origen extranjero en el centro educativo.

Este plan recoge el conjunto de actuaciones que el centro desarrolla para favorecer la acogida e inclusión del alumnado de origen extranjero y sus familias. Se perfila como un documento de centro donde recoger el qué y cómo de la atención a este alumnado.

Partiendo de la autonomía en la gestión de los centros educativos, estos establecerán las líneas de actuación en los documentos de organización del centro, basándose en la contextualización del centro, el entorno, el alumnado matriculado, etc. Esta planificación se establecerá para cada curso académico.

Este programa puede redactarse en un documento específico o transversalizarse en los documentos de organización del centro educativo (proyecto educativo, plan de convivencia del centro, plan de atención a la diversidad) y se basará en los principios generales del plan marco de acogida e inclusión (programa tres del dossier) atendiendo a las peculiaridades de cada centro.

Programa 3. Plan marco de acogida e inclusión al alumnado y las familias de origen extranjero en el sistema educativo

Este plan se considera el eje de vertebrador en el que se sustenta la planificación y organización de las actuaciones educativas. Se caracteriza por ser un documento generalista dentro de la organización y planificación de los centros educativos. 
Respecto al alumnado al que se refiere este trabajo, el documento recogerá los criterios generales de actuación en el proceso de incorporación y permanencia del alumnado de origen extranjero al sistema educativo, delimitando las diferencias en los distintos niveles. Será elaborado por la administración educativa competente en cada comunidad y establecerá las pautas generales de organización que se deben tener en cuenta para que cada centro educativo establezca los recursos y programas adaptados a su realidad.

Los objetivos que se pretenden con este plan marco son: garantizar una inclusión educativa de calidad para todo el alumnado de origen extranjero; facilitar el proceso de escolarización, inclusión y participación del alumnado y de sus familias en el entorno escolar y en la sociedad, y proponer las medidas necesarias para la adecuada escolarización del alumnado teniendo en cuenta las características y necesidades que presenten.

\section{Programa 4. Programa de apoyo lingüístico}

El objetivo de este programa es propiciar el aprendizaje de las competencias comunicativas y lingüísticas necesarias para la correcta inclusión y promoción en el sistema educativo. Las actividades propuestas deberán basarse, siempre que sea posible, en los contenidos curriculares abordados en el aula de referencia.

Así mismo este programa deberá desarrollarse, con carácter general, dentro del aula de referencia mediante fórmulas organizativas, agrupamientos (trabajo individual, pequeño grupo, gran grupo y atención individualizada para el alumnado que lo requiera por parte del profesor o de otros compañeros) y programas de intervención variados y flexibles.

Este tipo de programa debe ser entendido con carácter excepcional y temporal, adaptándolo a las necesidades específicas de cada alumno, previa evaluación de las mismas

\section{Programa 5. Tutor de acogida y/o coordinador/a intercultural}

A través de este programa se atenderán las necesidades específicas del alumnado de origen extranjero, sus familias, el resto del alumnado, la comunidad educativa, etc. Este profesional se ocupará de la formación en educación intercultural, siendo el responsable de la coordinación y gestión de todas las acciones relacionadas con el campo de la interculturalidad y la inclusión educativa.

Esta figura no debe entenderse como un agente solitario que asume todas las funciones, sino que trabajará en colaboración con el resto del profesorado, de tal manera que esta persona sea una figura de apoyo y referencia para que el resto del profesorado. La función de este profesional quedará recogida en plan de acogida. 


\section{Programa 6. Programa de enlace con el entorno}

Este programa se entiende como una red de apoyo a los centros por parte de la comunidad educativa, de forma que pueda establecerse una coordinación con los recursos y servicios de instituciones y organizaciones sociales que se encuentren próximos a los centros. Por tanto, se puede definir como una iniciativa abierta y de cooperación educativa que trata de dar una respuesta integrada y comunitaria.

\section{Programa 7. Programa de mediación intercultural}

La mediación intercultural es un proceso de acercamiento entre personas de distintas culturas a través de una tercera persona imparcial (el mediador). Ante todo, el mediador interviene, construye un lenguaje común entre las partes, despliega un papel activo y delicado para el cual la formación profesional adquiere un valor fundamental (Moreno Moreno, 2006).

A través de este programa, los centros educativos disponen de una persona que favorezca la convivencia entre el alumnado de diversas culturas, fomentando la cohesión social e integrando la escuela en la comunidad educativa. Este recurso constituirá un apoyo para el profesorado, los centros escolares, el alumnado, las familias y la comunidad educativa.

Programa 8. Programa para el conocimiento y mantenimiento de la lengua y cultura de origen del alumnado de procedencia extranjera

La base de este programa se encuentra en que tanto el alumnado de origen extranjero como el de procedencia no extranjera, no debe renunciar a su origen lingüístico y cultural. Por ello, este programa se abre al alumnado inmigrante como el resto de compañeros para acercar los rasgos culturales y la lengua de todas las personas presentes en el centro.

La planificación de este programa puede realizarse en horario escolar o extraescolar y puede contar con la colaboración de asociaciones o recursos de la comunidad educativa especialistas en este ámbito.

\section{Programa 9. Centros de recursos para trabajar la educación intercultural}

Este programa aportará apoyo técnico y didáctico en relación a la acogida e inclusión de la población de origen extranjero en el contexto educativo. Serán un servicio para la comunidad educativa y la sociedad en general.

La labor de estos centros de recursos es la de ofrecer fuentes bibliográficas especializadas (diccionarios, traducciones, asesoramiento en la realización de programas diversos para trabajar la educación intercultural y la acogida e integración del alumnado extranjero), materiales y recursos ya elaborados y puestos en práctica por centros educativos (en grupos de trabajo y formación en centros de trabajo). Todo ello generando un gran banco de documentación y recursos sobre interculturalidad, 
acogida e integración escolar, disponible para toda la comunidad educativa que, a través de este centro, puede coordinarse con los centros de profesorado.

\section{Programa 10. Alumnado mentor de acogida}

Este programa se desarrollará con la colaboración de los compañeros del aula en la que se matricule el alumno de origen extranjero. Se pretende cubrir las necesidades que puedan tener los recién llegados a partir de la cercanía y proximidad de un igual.

Este alumnado mentor constituirá durante un periodo inicial (una o dos semanas) el guía en los tiempos de ocio y relaciones interpersonales con los compañeros recién llegados, estableciendo relaciones entre el alumno y el profesor tutor de acogida. También puede acompañar al alumno y mostrarle cual es el aula de referencia y el aula de apoyo lingüístico (si precisa) y el resto de dependencias del centro.

\section{Programa 11. Servicio de traducción e interpretación}

Este servicio será el encargado de acortar la barrera lingüística que se interpone en el momento de la escolarización del alumnado y durante su posterior acogida en el centro. Este programa será el puente de comunicación e intercambio de información entre la administración, los centros, las familias y el alumnado.

Además de posibilitar la comunicación, también se encargará de la traducción de documentos relevantes para los centros escolares y las familias. Con estas traducciones se creará un archivo de documentación accesible a toda la comunidad educativa.

\section{Programa 12. Cartelería en las distintas dependencias del centro}

Este programa permitirá la ubicación de las diferentes aulas y estancias de los centros, facilitando la acogida de todo el alumnado. Estas indicaciones se realizarán en diferentes idiomas permitiendo la movilidad y el proceso de integración.

\section{Programa 13. Guía de información multilingüe del sistema educativo}

La función principal de este programa es facilitar al alumnado y las familias la comprensión de la estructura y organización del sistema educativo español. Su elaboración a través de un lenguaje claro y sencillo permitirá la integración de todos en la comunidad educativa.

Alguna de la información que deberá recoger este documento será: estructura del sistema, organización y tipos de centros, trámites de matrícula, programas específicos, becas, teléfonos y direcciones de interés, etc. 


\section{Conclusiones}

La sociedad actual es multicultural y diversa lo que ha generado la modificación de las estructuras sociales como respuesta a las necesidades surgidas de esta nueva realidad. Sin duda, los procesos migratorios son un hecho que ha protagonizado esta nueva visión de la sociedad.

Un hecho sin precedente que surge con la inmigración, es la llegada de menores al país de acogida, lo que provoca la escolarización de estos hijos de la inmigración en el sistema educativo. Ahora más que nunca, los colegios son lugares caracterizados por la heterogeneidad y la diversidad de rasgos culturales, religiosos, de formas de vestir, etc.

Este cambio social ha exigido el desarrollo de actuaciones educativas que han evolucionado desde perspectivas de compensación educativa, hacia la integración escolar, para finalmente desarrollar planteamientos de educación intercultural y de escuela inclusiva, en línea con las propuestas del contexto internacional (Booth y Ainscow, 2002; Ainscow, Booth y Dyson, 2006).

En este sentido, la educación inclusiva tiene como propósito prestar una atención educativa que favorezca el máximo desarrollo de todo el alumnado y la cohesión de los miembros de la comunidad, para ello se debe ofrecer al alumnado altas expectativas de éxito educativo independientemente de sus características, necesidades o discapacidades. La UNESCO (2009:8) considera que

"la educación inclusiva es un proceso de fortalecimiento de la capacidad del sistema educativo para llegar a todos los educandos".

Para la consecución de estos fines, el sistema escolar debe proveer los apoyos necesarios, para que cada alumno pueda seguir el ritmo del grupo.

En relación a las conclusiones que se extraen del estudio realizado y la elaboración del dossier para la inclusión educativa del alumnado de origen extranjero, a partir de la documentación, comentarios e información recibida por parte del panel de expertos, podemos considerar las siguientes apreciaciones.

La revisión de los programas por comunidades pone de manifiesto unas líneas comunes de actuación en todas ellas.

Los programas identificados pueden clasificarse en cuatro ítems: recursos destinados al alumnado, a las familias, al profesorado y a la comunidad educativa. Esta clasificación no es algo cerrado, ya que algunos programas se dirigen transversalmente a varios destinatarios.

El planteamiento general, en todos los casos, es el intento por incorporar los documentos y programas específicos para este alumnado dentro de los documentos generales del centro. Sólo cuando es estrictamente necesario se les da un carácter especializado. 
Los programas deben ser revisados y organizados por los docentes implicados e integrarlos en la comunidad educativa, además han de ser analizados anualmente. Estas propuestas deben ser medidas de carácter normalizador, integradas de forma natural en los centros escolares.

El dossier está compuesto por programas que se encuentran en distintos niveles de desarrollo en cuanto a la planificación educativa. Esto es, encontramos programas que establecen líneas marco dentro de la organización y planificación educativa (véase el programa de acogida y el plan marco). En otro nivel encontramos los programas que establecen pautas generales de actuación, como el de mediación intercultural, el servicio de interpretación y traducción, los centros de recursos, el programa de enlace y el plan de formación del profesorado.

Después tenemos programas que se desarrollan directamente en la escuela, como el tutor de acogida, el alumnado mentor, el programa de apoyo lingüístico, la guía de información multilingüe o la cartelería.

La familia y la comunidad educativa adquieren dentro de este dossier un lugar imprescindible, convirtiéndose en elementos fundamentales para la inclusión de este alumnado.

Apuntar también que este dossier nace del consenso de expertos, por lo que se puede establecer su validez para todas las comunidades, salvando las características propias de cada centro y la individualidad de las personas.

\section{Referencias bibliográficas}

AINSCOW, M; BOOTH, T y DYSON, A. (2006). "Inclusion and the standards agenda: negotiating policy pressures in England". En: International Journal of Inclusive Education, $\mathrm{n}^{\mathrm{o}} 10$, p. 295-308.

ALCALDE CAMPOS, R. (2008). "Los programas de actuación educativa orientados al alumnado de origen extranjero: modelos de atención a la diversidad cultural o a la igualdad educativa?" En: Revista de Educación, n³45, Madrid, p.207-228.

ARJONA, A. (et al.). (2008). Jóvenes inmigrados y educación en España. Variables que afectan en su incorporación a la Universidad de Almería: propuestas de actuación. Almería: Universidad de Almería.

AUSÍN, V. y LEZCANO, F. (2011). Recursos para la inclusión educativa de alumnado de origen extranjero en España: diseño y validación. Disponible en http://bit. 1y/19s8ODu. [10-10-2013].

BESALÚ, X. (2002). Diversidad cultural y educación. Madrid: Editorial Síntesis.

BOOTH, T. Y AINSCOW, M. (2000). Index for inclusion. Traducción castellana Guía para la Evaluación y Mejora de la Educación Inclusiva. Madrid: Consorcio Universitario parta la Educación Inclusiva. 
CARRASCO PONS, P. (2005). "Interculturalidad e inclusión: principios para evaluar la acogida al alumnado de origen extranjero". En: Revista Aula Innovación Educativa, ${ }^{\circ}$ 147, Murcia, p. 64-68.

CIDE. (2005). La atención al alumnado inmigrante en el sistema educativo en España. Madrid: Ministerio de Educación y Ciencia.

FERNÁNDEZ BATANERO, J. M. (2006). "Educación del alumnado inmigrante: propuestas educativas". En: Revista Iberoamericana de Educación, $\mathrm{n}^{\circ} 1$, Madrid. p. $1-10$.

FERNÁNDEZ FERNÁNDEZ, I. M. (2010). Atención a la diversidad y equiparación de oportunidades: una nueva mirada en la escuela inclusiva. Odiseo, revista electrónica de pedagogía, 7, (14). Disponible en http://www.odiseo.com. $\mathrm{mx} / 2010 / 7-14 /$ fernandez-atencion_diversidad.html. [29-09-2013].

JEFATURA DEL ESTADO. Ley Orgánica 2/2006 de 3 de Mayo, de Educación (LOE). Boletín Oficial del Estado, de 4 de Mayo de 2006, núm. 106, p. 17158 17207.

MORÁGUEZ IGLESIAS, A. (2006). ¿Cómo seleccionar el tamaño de una muestra para una investigación en educación? Disponible en http://bit.ly/Hr4paD. [2-102013].

MORALES OROZCO, L. (2006). La integración lingüistica del alumnado inmigrante. Propuestas para el aprendizaje cooperativo. Madrid: Los libros de la catarata.

MORENO MORENO, J. (2006). "La mediación en el ámbito de la inmigración y la convivencia intercultural". En: Acciones e investigaciones sociales, $\mathrm{n}^{\circ} 1$, Zaragoza, p. 1-35.

OLIVER VERA, M ${ }^{\mathrm{a}}$ C. (2003). Estrategias didácticas y organizativas ante la diversidad: dilemas del profesorado. Barcelona: Octaedro

RAMIREZ SERRANO, $\mathrm{M}^{\mathrm{a}}$ C. (2010). "Respuesta educativa ante las necesidades del alumnado inmigrante". En: Revista digital para profesionales de la enseñanza, $\mathrm{n}^{\circ} 10$, Andalucía, p. 1-9.

RUIZ OLABÚENAGA, J. I. (1999). Metodología de la investigación cualitativa. Bilbao: Universidad de Deusto

UNESCO (2009). Directrices sobre politicas de inclusión en la educación. Publicado por la Organización de las Naciones Unidas para la Educación, la Ciencia y la Cultura. París: UNESCO.

\section{La autora}

Vanesa Ausín Villaverde. Doctora en Ciencias de la Educación por la Universidad de Burgos. Actualmente desarrollo mi labor profesional dentro de la citada universidad en el área de didáctica y organización escolar. La docencia la imparto en los grados de maestros (infantil y primaria), pedagogía y máster sobre educación y sociedad inclusiva. Mis líneas de investigación son la inclusión educativa y las nuevas tecnologías aplicadas a la educación. 